\title{
Multimedia sobre ciencias de la información y la comunicación en bibliotecas y servicios universitarios: BIBLIORed 3.0
}

Multimedia on information and communication sciences in university libraries and services: BIBLIORed 3.0

\author{
Alfonso LóPEZ YEPES \\ Servicio Documentación Multimedia. Facultad Ciencias de la Información, \\ Universidad Complutense Madrid, España, alopez@ucm.es
}

\begin{abstract}
Resumen
Se describe y contextualiza Bibliored 3.0, un proyecto de investigación iniciado en octubre 2014 por el Servicio de Documentación Multimedia de la Universidad Complutense de Madrid. Bibliored 3.0 contribuye a difundir la producción multimedia propia y a facilitar el acceso a la producción ajena estrechamente relacionada sobre las ciencias de la información y comunicación, en bibliotecas y servicios especializados universitarios, reduciendo la dispersión informativa existente. Destaca por su interés el canal RTVDoc, en YouTube. Se recomienda la coordinación a través de servicios como "La Universidad Responde" del Servicio Audiovisual de Universidades Españolas.

Palabras clave: Acceso abierto. Bibliotecas universitarias. Ciencias de la información. Ciencias de la comunicación. Iberoamérica. Multimedia. Servicio Universitario. Universidad Complutense de Madrid. Bibliored 3.0.
\end{abstract}

\section{Introdución}

El Servicio de Documentación Multimedia (1993-2015) de la Universidad Complutense de Madrid (dependiente del Departamento de Biblioteconomía y Documentación) viene desarrollando actividades académico-científicas desde 1993, materializadas en proyectos de investigación y otras actuaciones generadoras de producción multimedia propia en el ámbito de la documentación informativa.

Su propuesta más reciente, desde noviembre de 2014, ha sido el establecimiento de un sitio que integra y remite a contenidos en estrecha relación con la información y documentación multimedia sobre ciencias de la información y comunicación en bibliotecas y servicios especializados universitarios, en acceso abierto y proyección iberoamericana. Dicha web, denominada Bibliored3.0, canaliza tanto producción propia como acceso a producción ajena relacionada con el tema, en la idea de convertirse en un repositorio multimedia de necesario mantenimiento que cubra en lo posible la dispersión y proporcione constantemente información pertinente para in-

\begin{abstract}
Bibliored 3.0 is a research project started in October 2014 by the Multimedia Documentation Service of the Complutense University of Madrid. Bibliored 3.0 aims at disseminating the multimedia production of Iberoamerican universities and other closely related multimedia content on information and communication sciences, in university libraries and specialized documentation services. One of its star productos is the RTVDoc channel on YouTube. It is recommended that universities improve the dissemination of their productions through coordination services like "The University Answers" of the Spanish Universities Audiovisual Services.
\end{abstract}

Keywords: Open Access. Communication sciences. Iberoamerica. Information sciences. Multimedia. University libraries. University services. Universidad Complutense de Madrid. Bibliored 3.0.

vestigaciones pluridisciplinares. Información dirigida y multidisciplinariedad representada por archiveros, bibliotecarios, documentalistas, investigadores en general, comunicadores en general, periodistas, cineastas, publicitarios, sociólogos, literatos, psicólogos, antropólogos, filósofos...y a un amplio número de profesionales de todo tipo de conocimiento. En muy estrecha relación con los medios de comunicación social, en la idea asimismo de que la biblioteca es en la actualidad también un medio de comunicación -Biblioteca 2.0-, un ámbito informativo y también de producción propia (Bibliored3.0, 2015).

\section{Método}

La revisión de la literatura relacionada con el tema nos ha permitido establecer una serie de puntualizaciones que han propiciado la configuración en el ámbito del servicio de documentación multimedia de la UCM de un sitio, específico: Bibliored3.0. Con objeto de que canalice todo ello al mismo tiempo, que reduzca la dispersión informativa existente, con la filosofía de repositorio multimedia, que clasifique pertinen- 
temente conocimiento especializado. Internet ha transformado el concepto y el uso de la información, dotando de nuevos roles al documentalista o gestor de información digital. Al bibliotecario se le considera también documentalista con todo lo que ello conlleva en cuanto a nuevo profesional de la información, y en ese sentido el bibliotecario también es gestor de información, analista de información, experto en usabilidad y hasta incluso diseñador conceptual multimedia (López Yepes, J., 2015).Y la biblioteca medio de comunicación que produce y difunde contenidos (López Yepes, A., 2014).

La información audiovisual y multimedia en bibliotecas universitarias está presente en blogs, boletines electrónicos, e-prints, revistas multimedia, canales IPTV y portales propios, en redes sociales y tabletas y teléfonos inteligentes. Existen radios y televisiones universitarias y servicios audiovisuales vinculados a las universidades: Servei d'Audiovisuals UB (Servei
d'Audiovisuals UB, 2015), Servei de Recursos Audiovisuals UIB (Servei Recursos Audiovisuals UIB, 2015), Complumedia o el propio Servicio de Documentación Multimedia de la UCM. O también Planeta Biblioteca canal radiofónico creado por Bibliotecas de la Universidad de Salamanca, con 32.500 descargas en agosto 2015 (Planeta Biblioteca, 2015). O BiblioTV, de la Dirección General Bibliotecas Benemérita Universidad Autónoma Puebla-México (BiblioTV, 2013). Y los webinars, hangouts de google, youtube, skype, livestream, ustream, y otros sistemas streaming (IIBI, 2015). Y actuaciones como "La Universidad Responde" (Servicios Audiovisuales de las Universidades Españolas) (SAVUES, 2012), a las que deben acogerse con más presencia las bibliotecas universitarias. Y, en fin, para su aplicación en el ámbito de las bibliotecas universitarias deben contemplarse las nuevas tendencias como los transmedia y la distribución multiplataforma (Díaz, Tous, 2012).

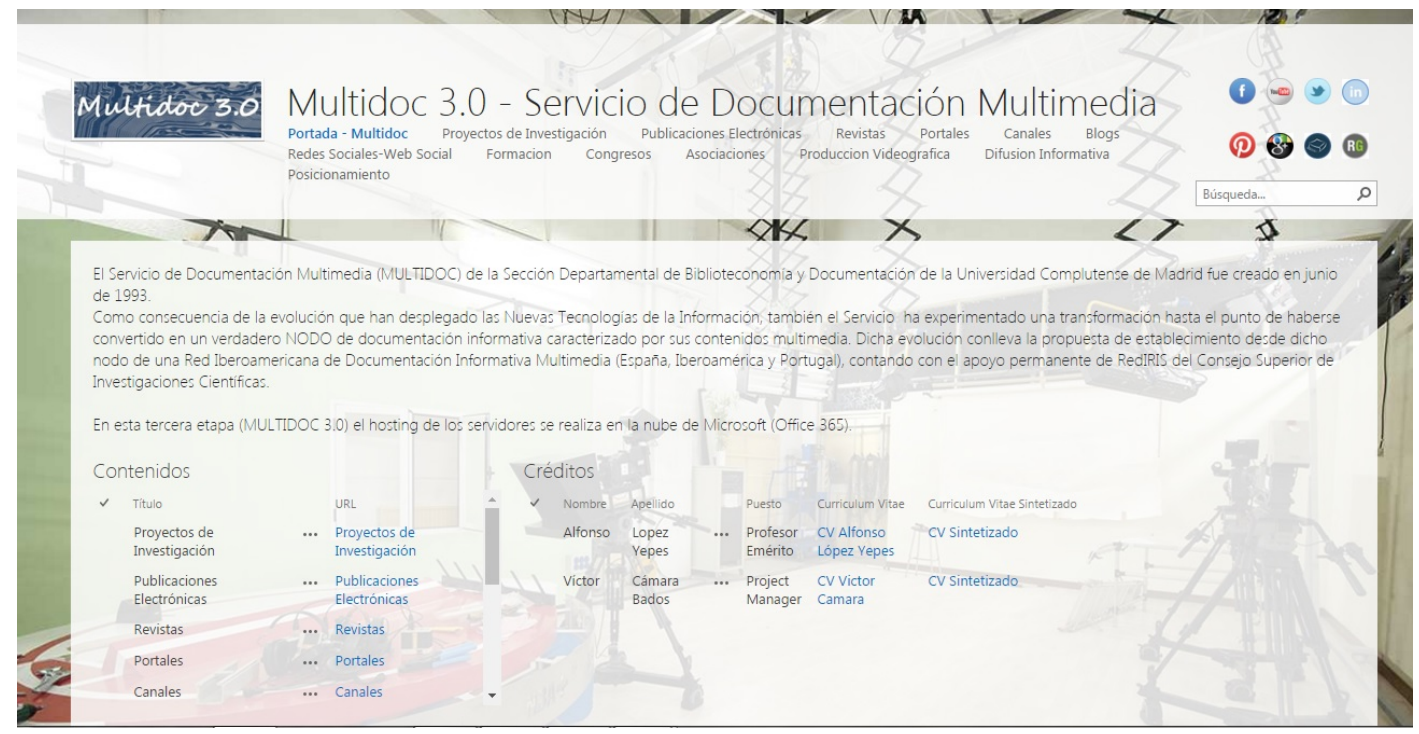

Figura 1. Servicio de Documentación Multimedia

\section{Resultados: Bibliored $\mathbf{3 . 0}$}

Como consecuencia de la situación planteada, previa revisión de la literatura científica como respuesta al estado de la cuestión, se configuró en octubre de 2014 una herramienta estándar en forma de blog-portal en el ámbito del Servicio de Documentación Multimedia.

Bibliored 3.0 se administra y mantiene en el Servicio de Documentación Multimedia del Dpto. de Biblioteconomía y Documentación de la UCM. Dispone de dominio propio e ISSN 23868880, asignado por la Biblioteca Nacional de España (BNE, 2015) con fecha 26.01.2015, e integra y remite desde su página de bienvenida a otras realizaciones de producción propia de creación anterior sobre documentación informativa. Como consecuencia de este planteamiento (entroncado con los medios de comunicación que en realidad transmiten y proyectan todo el conocimiento humano), Bibliored 3.0 canaliza conocimiento multidisciplinar de interés por su diversidad para usuarios reales y potenciales, de prácticamente cualquier especialidad (bibliotecarios, comunicadores, sociólogos, psicólogos, literatos, historiadores, investigadores...), sustentado en todo momento en ámbitos de almacenamiento, producción y difusión multimedia materializados en bibliotecas, filmotecas, fonotecas, fototecas, videotecas, mediatecas... 


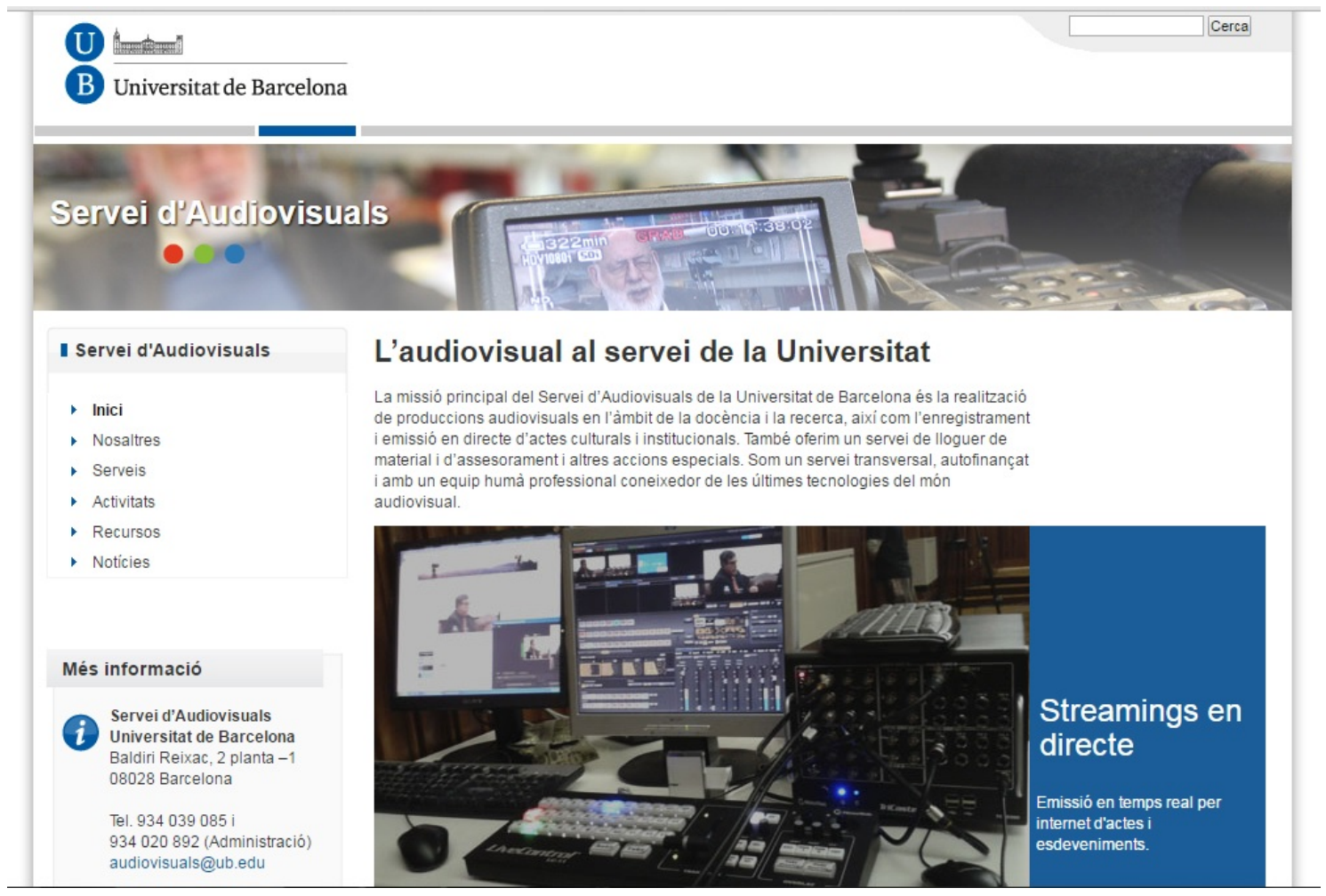

Figura 2. Servei d'Audiovisuals UB

El sitio presenta sus contenidos clasificados en cuatro apartados que conforman su estructura: un menú horizontal con páginas y subpáginas de contenidos; la parte central del sitio para el desarrollo de los post; un menú lateral izquierdo para las etiquetas y ámbitos multimedia en forma de instrumentos que facilitan el acceso a otros documentos asociados (videotecas, fototecas, fonotecas, canales, portales) y a proyectos previos desarrollados en el marco del servicio de documentación multimedia. La producción propia de contenidos multimedia y su difusión se lleva a cabo a través del canal RTVDoc desde 2008 (RTVDoc, 2015) realizándose la recuperación de dichos contenidos mediante etiquetas y búsqueda avanzada (los documentos están accesibles a través de cualquier palabra contenida en ellos). Se posibilita el acceso a temáticas diversas: metodología, web semántica, patrimonio, hispana (europeana), Iberoamérica. $Y$ el menú lateral derecho está destinado a espacios para la recuperación documental, estadísticas de acceso, recursos informativos relacionados con los contenidos publicados, documentos más consultados, modos de suscripción al sitio, estadísticas de seguidores a través de redes sociales, licencia, acceso a conocidas herramientas de producción y recuperación de referencias como Mendeley o Scoop-it y el mapa ciberespacial de visitantes.
Por otra parte, similitudes, diferencias, puntos fuertes, puntos débiles... de Bibliorted3.0 en comparación con otros sitios ya existentes con anterioridad y recién aludidos en este mismo epígrafe, se resumen en los siguientes puntos:

- ISSN 2386-8880 (punto fuerte).

- Vídeo de presentación de elaboración propia con descripción de contenidos (punto fuerte).

- Producción propia y acceso a producción de otros ámbitos estrechamente relacionados temáticamente (similitud con otros servicios).

- El sitio canaliza conocimiento multidisciplinar, de interés por su diversidad para usuarios reales y potenciales, de prácticamente cualquier especialidad: bibliotecarios, comunicadores, sociólogos, psicólogos, literatos, historiadores, investigadores... Aporta un conocimiento más amplio y multidisciplinar.

- Conocimiento sustentado en ámbitos de almacenamiento, producción y difusión multimedia materializados en bibliotecas, filmotecas, fonotecas, fototecas, videotecas, mediatecas... Remite a todo tipo de archivos y ámbitos especializados de almacenamiento, conservación, recuperación, preservación, difusión del patrimonio audiovisual-multimedia... en y para bibliotecas y servicios especializados universitarios (punto fuerte). 
- Integra-incorpora acceso a un canal IPTV propio (RTVDoc), integrado en Complumedia UCM y también en YouTube (punto fuerte).

- Recuperación de contenidos mediante etiquetas y búsqueda avanzada (los documentos están accesibles a través de cualquier palabra contenida en ellos), estadísticas de acceso, recursos informativos relacionados con los contenidos publicados, documentos más consultados, modos de suscripción al sitio, estadísticas de seguidores a través de redes sociales, licencia, acceso a conocidas herramientas de producción y recuperación de referencias como Mendeley o Scoop-it y el mapa ciberespacial de visitantes. Ofrece similitudes con otros sitios, pero también diferencias en cuanto a reunir conjuntamente varias herramientas muy difundidas en la actualidad sobre difusión multimedia.

- Convendría integrar en la web la posibilidad de efectuar emisiones radiofónicas en la lí- nea por ejemplo del canal radiofónico Planeta Biblioteca, creado en el ámbito bibliotecario de la Universidad de Salamanca (punto débil).

En fin, se remite a algunos ejemplos de difusión del sitio a través de blogs, portales y redes sociales: Biblogtecarios (Biblogtecarios, 2015), Biblogsfera (Biblogsfera, 2014), El documentalista audiovisual (EDA, 2015) o Universo Abierto (Universo Abierto, 2015). O a través de los EPrints Complutense (López Yepes, A. 2007), también accesibles vía REBIUN y su directorio de repositorios (REBIUN, 2015). Y en redes sociales tanto de ámbito general con el establecimiento de grupos en Facebook como Audiovisual 3.0 o como Somos 2.0 (Somos 2.0, 2015), Audiovisual 3.0 (Audiovisual 3.0) o Multimediabolivia (Multimediabolivia, 2015). Y otras redes sociales: Twitter (Twitter, 2015), Academia.edu (Academia, 2015), Researchgate.net (Researchgate, 2015), Linkedin (Linkedin, 2015) o Cero en conducta (Cero en conducta, 2015).



Figura 3: Bibliored3.0

\section{Discusión}

A pesar de la difusión informativa realizada sobre Bibliored 3.0 en ámbitos profesionales especializados, no se ha provocado una intensa discusión en dichos medios. Disponemos de unas pocas referencias sobre el tema: la planteada por Javier Leiva el 27.09.2015 (Noticias ANABAD y ThinkEPI) y comentada por el autor de estas páginas el 2.03.2015 (Leiva, 2015; López Yepes, A., 2015). También motivo para el debate sobre patrimonio audiovisual-multimedia, acceso abierto y proyección iberoamericana supuso en junio 2015 el IV Seminario Hispano
Brasileño de Investigación en Información, Documentación y Sociedad (Marília-Brasil): grupo de trabajo sobre conservación, preservación, restauración hispano brasileña en el marco de Bibliored 3.0, a propósito de las actuaciones brasileñas CRIDI y Legatum (CRIDI, 2015; Legatum, 2015; López Yepes, A., 2015b).

Y la propuesta de una "Red universitaria de patrimonio multimedia en bibliotecas y servicios universitarios en acceso abierto y proyección iberoamericana". Dicho proyecto presentaría una estructura y sistematización de contenidos en esta línea o parecida: a) temáticas, tipologías 
y ámbitos de aplicación —vídeos sobre bibliotecas y servicios universitarios e instituciones estrechamente relacionadas-; b) diseño de herramientas-instrumentos para producción audiovisual-multimedia propia y acceso a pro- ducción de otras instituciones relacionadas; c) difusión informativa - presencia en redes sociales generales y especializadas-; y d) publicaciones especializadas: blogs, portales, redes...plataformas virtuales iberoamericanas.

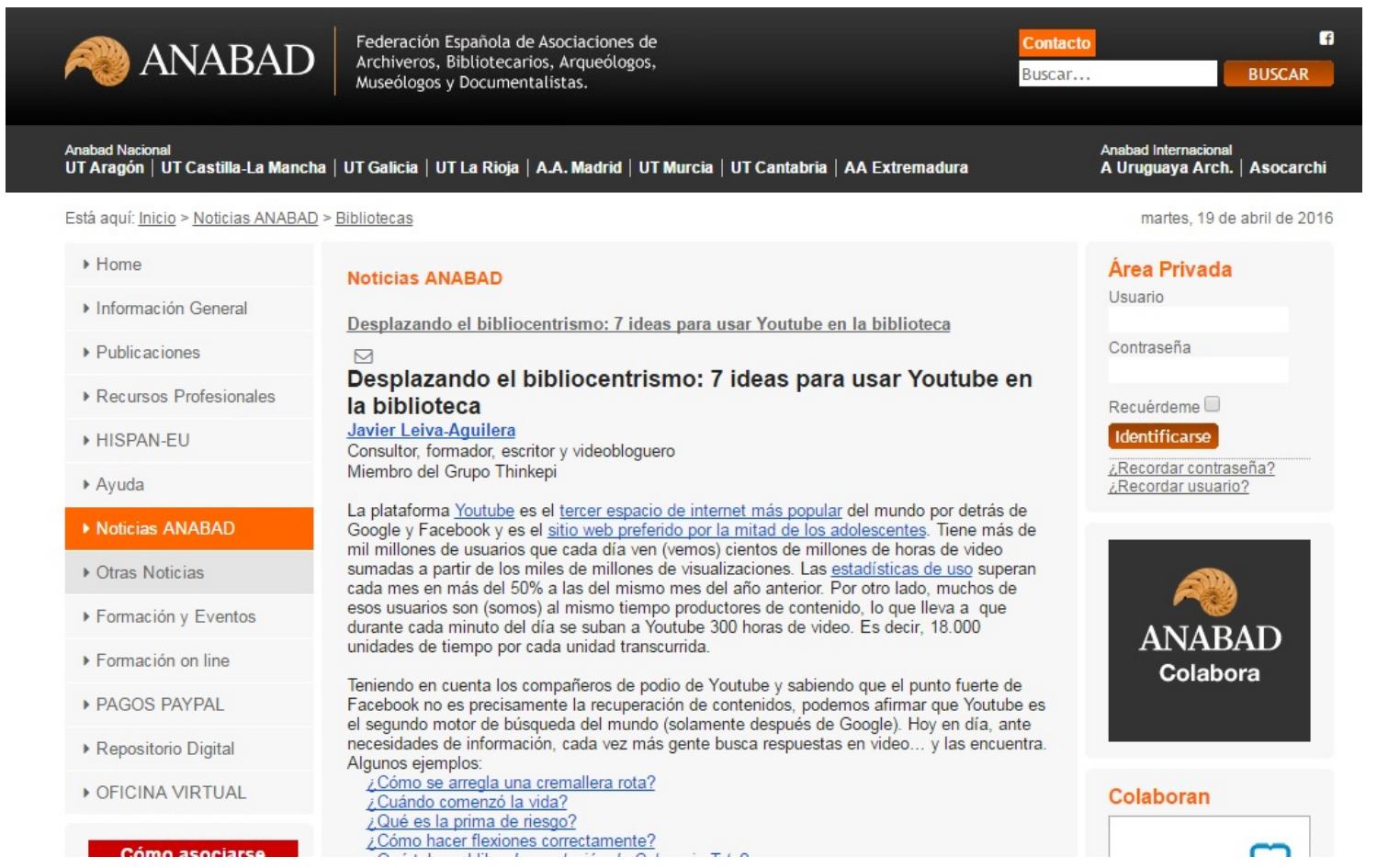

Figura 5. ANABAD-ThinkEPI

\section{Conclusiones}

La consulta y revisión de fuentes especializadas manifiestan carencia de bibliografía exclusiva sobre los siguientes ámbitos temáticos relacionados entre sí: multimedia, ciencias de la información y comunicación, bibliotecas y servicios universitarios, acceso abierto y proyección iberoamericana. Aunque existen iniciativas en España como SAVUES (Servicios Audiovisuales de Universidades Españolas), así como radios y televisiones universitarias apenas si aparece reflejada en dichos ámbitos universitarios la presencia y actividades de bibliotecas y servicios universitarios.

Se utilizan plataformas y sistemas para la producción, recuperación y difusión de contenidos multimedia: webinars, hangouts, canales youtube, livestream, ustream, skype... Aunque lo más adecuado sería que bibliotecas y servicios universitarios hicieran uso de configuraciones propias como RTVDoc y BiblioTV. Además, la información y producción multimedia en bibliotecas y servicios universitarios está presente, de forma dispersa y no muy generalizada, en blogs, boletines electrónicos, e-prints, revistas multimedia, canales IPTV y portales propios, en redes sociales y tabletas y teléfonos inteligentes.

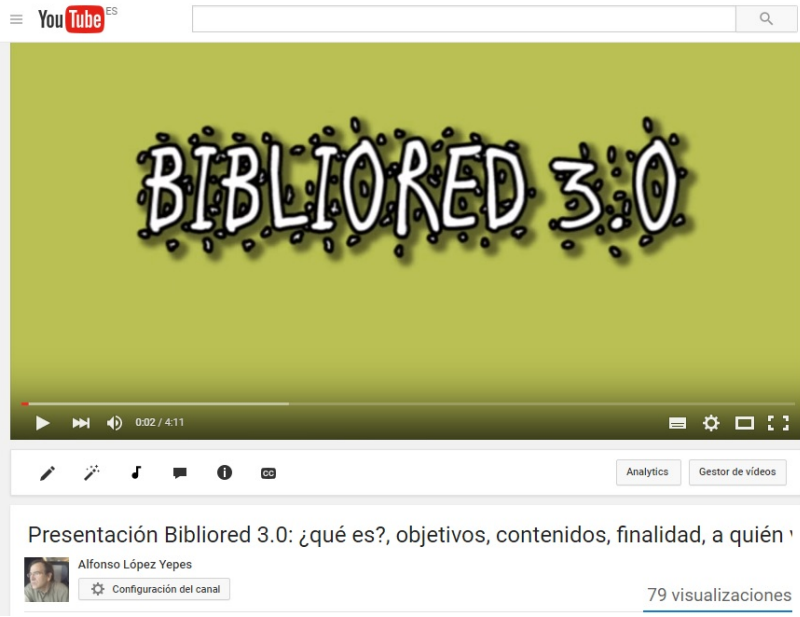

Figura 4: Bibliored3.0 vídeo presentación

El debate sobre el estado de la cuestión es escaso. BIBLIOred 3.0 supone provocar la discusión en torno al tema y el establecimiento de 
una web para localizar, integrar y difundir en acceso abierto y proyección iberoamericana información pertinente, contribuyendo con ello a contrarrestar la dispersión existente.

\section{Referencias}

Academia.edu (2015). Red social académico-científica https://www.academia.edu/11828086/Bibliored_3.0_mult imedia_sobre_ciencias_de_la_informaci\%C3\%B3n-com unicaci\%C3\%B3n en bibliotecas-servicios universitari os_(2015.09.23).

Audiovisual 3.0 (2015). Grupo de trabajo en Facebook. https://www.facebook.com/groups/audiovisual3.0/ (2015.09.23)

Bibliocineradiotv (2015). Grupo de trabajo en Facebook. https://www.facebook.com/groups/1390973431230112/ (2015.09.23).

Bibliored3.0 (2015). Multimedia sobre ciencias de la información en bibliotecas y servicios universitarios. http://www.bibliored30.com (2015.09.23).

BiblioTV (2013): Dirección General de Bibliotecas. Benemérita Universidad Autónoma de Puebla (Estado de México). https://www.youtube.com/watch?v=4QkJvnxEx g https://www.facebook.com/BiblioTV-137104169704473/ timeline/ (2015.09.23).

Bibliocineradiotv (2015). Grupo en Facebook en torno a "Multimedia sobre ciencias de la información y comunicación en bibliotecas y servicios de información y documentación universitarios". https://www.facebook.com/gro ups/1390973431230112/ (2015.09.23).

Biblogsfera (2014) Directorio de blogs relacionados com Biblioteconomía y Documentación:http://biblogsfera. com (2015.09.23).

Biblogtecarios (2015). Blog colaborativo de Biblioteconomía y Documentación. http://www.biblogtecarios.es/firmas invitadas/bibliored-3-0-multimedia-sobre-ciencias-de-la-i nformacion-comunicacion-en-bibliotecas-servicios-univer sitarios/ (2015.09.23).

BNE Depósito Legal (2015). http://www.bne.es/es/Colec ciones/Adquisiciones/DepositoLegal/

http://www.bne.es/es/LaBNE/CentroEspanolISSN/SolicitudIS $\mathrm{SN} /(2015.09 .23)$

Cero en Conducta-Educación y Cine (2014). Grupo de trabajo sobre documentación cinematográfica. http://ceroenconducta.ning.com/group/documentacioncinematografica (2015.09.23).

Díaz Noci, J.; Tous Rovirosa, A. (2012). La audiencia como autor: narrativas transmedia y propiedad intelectual del público. // El profesional de la información, septiembreoctubre. 21, 458-467.

EDA (2014) Blog El documentalista audiovisual. // Bibliored 3.0. http://eldocumentalistaudiovisual.com/?s=bibliored+ 3.013 (2015.09.23).

IIBI (2015). Livestream y Ustream para videoconferencia: http://original.livestream.com/iibiunam http://www.ustrea m.tv/channel/cuib-unam (2015.09.23).

Legatum (2015) Proyecto del Grupo de Estudos sobre Cultura, Representaçâo e Informaçâo Digitais-CRIDI: Repositorio: http://www.cridi.ici.ufba.br/?page_id=489 (2015.09. 23).

Leiva Aguilera, Javier (2015). Desplazando el bibliocentrismo: 7 ideas para usar Youtube en la biblioteca. // Noticias ANABAD: http://www.anabad.org/noticias-anabad/ 28-bibliotecas/3515-desplazando-el-bibliocentrismo-7ideas-para-usar-youtube-en-la-biblioteca. (2015.09.23). Anuario ThinkEPI, Vol.9, 2015, págs.78 y 79.
Linkedin (2014) Grupo de debate en Linkedin: https://www.linkedin.com/grp/post/3004310-5966540910 945660929?goback=\%2Egna_3004310 (2015.09.23).

López Yepes, Alfonso (2014). Audiovisual en línea en la universidad española: bibliotecas y servicios especializados (una panorámica). // Icono 14. 12:2. http://www .icono14.net/ojs/index.php/icono14/article/view/689 (2015.09.23).

López Yepes, Alfonso (2015a). A propósito de desplazando el bibliocentrismo: 7 ideas para usar Youtube en la biblioteca. // Anuario ThinkEPI.9, 79. https://www.research gate.net/publication/277921208_Desplazando_el_biblioc entrismo_7_ideas_para_usar_YouTube_en_la_bibliotec a (2015.09.23).

López Yepes, Alfonso (2015b). Patrimonio audiovisual en bibliotecas y servicios universitarios a propósito del servicio de documentación multimedia de la universidad complutense. Brasilia: Universidad Nacional (UnB) (En prensa).

López Yepes, José (2015). La ciencia de la información documental. // El documento, la disciplina y el profesional en la era digital. México D.F., Universidad Panamericana.

Multimediabolivia (2014). Grupo en Facebook: https://www.facebook.com/groups/Multimediabolivia3.0/ (2015.09.23).

Peset, F.; Ferrer Sapena, A.; Subirats Coll, I. (2011). Open data y Linked open data: su impacto en el área de bibliotecas y documentación. // El profesional de la información. 20:2, 165-173.

Planeta Biblioteca (2015). Temáticas radiofónicas sobre Biblioteconomía y Documentación.https://www.facebook .com/planetabibloioteca (2015.09.23).

REBIUN (2015). Directorio de repositorios: repositorio UCM, E-prints Complutense. http://www.rebiun.org/reposito rios/Paginas/Directorio-de-Repositorios-InstitucionalesREBIUN.aspx http://roar.eprints.org/463/ (2015.09.23).

Researchgate.net (2015). Bibliored 3.0: https://www.re searchgate.net/publication/273462819_Bibliored_3.0_m ultimedia_sobre_ciencias_de_la_informacin-comunicaci $\mathrm{n}$ en bibliotecas-servicios universitarios \%28\%2A $\% 29$ (2015.09.23).

RTVDoc (2015). Canal Servicio Documentación Multimedia en YouTube. http://www.youtube.com/user/alyepes1. Con subcanales-listas reproducción: https://www.you tube.com/user/alyepes1/playlists (2015.09.23).

SAVUES (2012). III Jornadas Servicios Audiovisuales Universidades Españolas: Escuelas Pías UNED 2728.09.2012. Madrid: UNED: https://canal.uned.es/serial/ index/id/408 15 (2015.09.23).

Servei de Recursos Audiovisuals UIB (2015). Universitat Illes Balears. http://srav.uib.cat/ (2015.09.23).

Servei d'Audiovisuals de la UB (2015). Universitat de Barcelona. http://www.ub.edu/audiovisuals/laudiovisual-al-ser vei-de-la-universitat (2015.09.23).

Somos 2.0 (2015). Grupo Facebook coordinado por Julio Alonso Arévalo. https://www.facebook.com/groups/somo s20/ (2015.09.23).

Twitter (2015). Referencia a Bibliored 3.0. https://twitter.com/ alyepes/status/573784057198284800 (2015.09.23).

Universo Abierto (2014). Blog biblioteca Facultad Traducción y Documentación Universidad de Salamanca. http://www .universoabierto.com/?s=bibliored+3.0 (2015.09.23).

Enviado: 2015-10-14. Segunda versión: 2016-04-29. Aceptado: 2016-04-29. 